\title{
Level Crossing Rates for MIMO Channel Eigenvalues: Implications for Adaptive Systems
}

\author{
Peter J. Smith Ping-Heng Kuo Lee M. Garth \\ Department of Electrical and Computer Engineering, University of Canterbury, Christchurch, New Zealand \\ \{p.smith,phk15,1.garth\}@elec.canterbury.ac.nz
}

\begin{abstract}
MIMO systems using adaptive transmission down the eigenchannels require the level crossing rate of the eigenvalues to compute adaptation rates and possibly feedback rates. Examples of such systems include MIMO systems using singular value decomposition (SVD) transmission. Similarly, the average fade durations of the eigenvalues give the average length of time that a particular constellation is used in adaptive modulation. Other systems which require the minimum eigenvalue to be above a certain threshold (for example, channel inversion) can also use these level crossing rates in system analysis. Hence, in this paper we derive approximate level crossing rates for the eigenvalues in the baseline case of an uncorrelated Rayleigh fading channel. Our results are remarkably accurate and compact and further improvements are also discussed.
\end{abstract}

\section{INTRODUCTION}

In recent years, Multiple Input Multiple Output (MIMO) systems have received considerable attention as they have the potential to provide quantum leaps in capacity [1]. To approach the high performance offered by MIMO systems, various transmitter and receiver processing methods have been proposed. The common goal is to use maximally the wireless link while the time-varying channel is favorable. For example, adaptive SVD techniques use larger constellations for eigenchannels with higher power [2]. Such techniques switch from one constellation to another as the channel eigenvalues cross certain thresholds. Hence, the level crossing rates (LCRs) of the eigenvalues give the adaptation rate of such a system. Similarly, the average fade durations (AFDs) of the eigenvalues give the average length of time that a particular constellation is used in adaptive modulation [3]. Another MIMO technique that depends on the eigenvalue levels is channel inversion. The minimum eigenvalue causes transmit power problems for channel inversion when it drops below a certain threshold [4]. Again, the LCRs of the eigenvalues give information about the performance of this technique. Further applications are discussed in [5] where the related issue of the joint time-varying behaviour of the channel matrix entries is considered. Hence, in this paper we derive a compact, closed-form approximation to the LCR of MIMO eigenvalues in the baseline case of an independent identically distributed (i.i.d.) Rayleigh fading channel. We also give the AFDs of the eigenvalues, the periods of time for which the eigenvalues remain below a threshold. Our results are very accurate for small to moderate systems, and further improvements are possible for larger systems.
TABLE I

Eigenvalue Means

\begin{tabular}{c|cccc}
\hline System & $\lambda_{1}$ & $\lambda_{2}$ & $\lambda_{3}$ & $\lambda_{4}$ \\
\hline \hline$(2,2)$ & 3.5 & 0.5 & & \\
\hline$(2,4)$ & 6.1875 & 1.8125 & & \\
\hline$(3,3)$ & 6.5208 & 2.1458 & 0.3333 & \\
\hline$(4,4)$ & 9.7723 & 4.4086 & 1.5692 & 0.25 \\
\hline
\end{tabular}

\section{Mimo Channel Model and Static Eigenvalue STATISTICS}

In this paper we assume a $\left(n_{T}, n_{R}\right)$ MIMO system with $n_{T}$ transmit and $n_{R}$ receive antennas. The channel matrix $\boldsymbol{H}$ is an $n_{R} \times n_{T}$ complex channel gain matrix containing i.i.d. complex Gaussian entries with unit magnitude variance. Let $m=\min \left(n_{R}, n_{T}\right), n=\max \left(n_{R}, n_{T}\right)$, and the $m \times m$ matrix $\boldsymbol{W}$ be given by

$$
\boldsymbol{W}= \begin{cases}\boldsymbol{H} \boldsymbol{H}^{\dagger}, & \text { for } n_{R} \leq n_{T} \\ \boldsymbol{H}^{\dagger} \boldsymbol{H}, & \text { for } n_{T}<n_{R}\end{cases}
$$

where $\dagger$ represents the conjugate transpose. Denoting the ordered eigenvalues of $\boldsymbol{W}$ by $\lambda_{1} \geq \ldots \geq \lambda_{m}$, the MIMO channel can be decomposed into $m$ parallel links [1] with powers given by $\lambda_{1}, \ldots, \lambda_{m}$. Matrix $\boldsymbol{W}$ is Wishart distributed, and the joint density of its eigenvalues is [1]

$$
\begin{aligned}
f\left(\lambda_{1}, \ldots, \lambda_{m}\right)= & \prod_{i=1}^{m}[(n-i) !(m-i) !]^{-1} \exp \left(-\sum_{i=1}^{m} \lambda_{i}\right) \\
& \times \prod_{i=1}^{m} \lambda_{i}^{n-m} \prod_{i<j}\left(\lambda_{i}-\lambda_{j}\right)^{2} .
\end{aligned}
$$

Since (2) is simply a polynomial with exponential terms, it can be integrated to yield the marginal densities of the eigenvalues. Expressions become rather cumbersome for $m \geq$ 4 , so a symbolic manipulation package is of considerable utility. Since the marginal densities themselves contain only polynomial and exponential terms, the mean and variance of the eigenvalues can be computed exactly. Values are given in Tables I and II for $(2,2),(2,4),(3,3)$ and $(4,4)$ systems. Similarly, the densities can be integrated to give the cumulative distribution functions (cdfs) of the eigenvalues. As an example, the $(2,2)$ system has eigenvalue densities $(f(\lambda))$ and cdfs 
TABLE II

EIGENVALUE VARIANCES

\begin{tabular}{c|cccc}
\hline System & $\lambda_{1}$ & $\lambda_{2}$ & $\lambda_{3}$ & $\lambda_{4}$ \\
\hline \hline$(2,2)$ & 3.25 & 0.25 & & \\
\hline$(2,4)$ & 5.4023 & 1.0273 & & \\
\hline$(3,3)$ & 5.5135 & 1.1385 & 0.1111 & \\
\hline$(4,4)$ & 7.6392 & 2.2442 & 0.5964 & 0.0625 \\
\hline
\end{tabular}

$(F(\lambda))$ given by

$$
\begin{aligned}
f\left(\lambda_{1}\right) & =\left(2-2 \lambda_{1}+\lambda_{1}^{2}\right) \exp \left(-\lambda_{1}\right)-2 \exp \left(-2 \lambda_{1}\right) \\
F\left(\lambda_{1}\right) & =1+\exp \left(-2 \lambda_{1}\right)-2 \exp \left(-\lambda_{1}\right)-\lambda_{1}^{2} \exp \left(-\lambda_{1}\right) \\
f\left(\lambda_{2}\right) & =2 \exp \left(-2 \lambda_{2}\right) \\
F\left(\lambda_{2}\right) & =1-\exp \left(-2 \lambda_{2}\right),
\end{aligned}
$$

where $\lambda_{1}, \lambda_{2} \geq 0$. The eigenvalue densities and cdfs for larger systems can be similarly derived.

Hence, we can see that the static behavior of the eigenvalues is known in great detail. Conversely, the behavior of the eigenvalues as the $\boldsymbol{H}$ matrix evolves over time appears to be virtually unknown, with the exception of the work by Bru [6] on Brownian motion matrices. When the temporal behavior of $\boldsymbol{H}$ is of interest we denote the matrix process by $\boldsymbol{H}(t)$ and its eigenvalues by $\lambda_{i}(t)$. In this paper, we propose to study the eigenvalue LCRs and AFDs as the elements of the $\boldsymbol{H}(t)$ matrix follow i.i.d. Jakes processes. Hence, each element is i.i.d. and shares a common autocorrelation function (ACF) given by $J_{0}\left(2 \pi f_{D} \tau\right)$ where $f_{D}$ is the Doppler frequency.

Note that the choice of the Jakes process is not required for the analysis, but for simplicity of exposition we keep with this widely used model throughout. Such a model is not handled in [6] which is limited to channel gains evolving as Brownian motion processes. This Brownian motion evolution is physically unreasonable as it implies a continuous increase in the magnitude variance of the channel gains over time. Nevertheless, we are able to leverage some of the results in [6] to complete our analysis.

\section{LEVEl CRossing RATES}

In principle, the exact LCRs for $\boldsymbol{\lambda}(t)=\left(\lambda_{1}(t), \ldots, \lambda_{m}(t)\right)$ can be computed from the joint density of $\boldsymbol{\lambda}(t)$ at times $t$ and $t+\tau$ where $\tau \rightarrow 0$. This joint density has been derived in [7], but the resulting expression is complex and would lead to multiple numerical integrals to evaluate the LCRs. Hence, we take an alternative approach and approximate the eigenvalue process by a gamma process. The accuracy of a gamma approximation to the eigenvalue density was pointed out in [8], and we take this a stage further by approximating the entire eigenvalue process by a gamma process. In Fig. 1 we show the exact distribution functions of $\left(\lambda_{1}, \ldots, \lambda_{4}\right)$ for a $(4,4)$ system with the fitted gamma distribution functions (obtained by equating the first and second moments). Agreement is excellent. Note that the smallest eigenvalue $\lambda_{m}$ has an exponential distribution for symmetric $(m, m)$ systems [9], which is a special case of the gamma distribution. Unfortunately, the accuracy of the gamma approximation decreases for larger systems, but a solution to this problem is discussed later. Also, fitting by the first two moments may not be optimal. Nevertheless, for all systems with $n \leq 4$ agreement is very good, so we adopt this simple method. Preliminary results show that the correlated Rayleigh and Ricean channels have eigenvalues which can also be approximated by gamma processes. Hence, a similar analysis may also be possible for a wider range of channel models, which is beyond the scope of this paper.

To discuss the gamma approximations, we need the following notation. Let a gamma distribution with shape parameter $r$ and scale parameter $\theta$ be denoted $G(r, \theta)$. Such a variable has mean and variance given by $r / \theta$ and $r / \theta^{2}$ respectively and pdf

$$
f(x)=\Gamma(r)^{-1} \theta^{r} x^{r-1} \exp (-\theta x), \quad x \geq 0 .
$$

Some work has appeared on gamma processes [10], but LCRs do not appear to be available. However, for the special case of a chi-squared $\left(\chi^{2}\right)$ process, the LCR is known [11]. This motivates us to represent a gamma process as an infinite (scale) mixture of $\chi^{2}$ processes. Note that a $\chi^{2}$ variable is simply $G(k, \theta)$ for integer shape parameter $k$. To construct this mixture, we extend the work of [12] on exponential variables to the current case and find that any $G(r, \theta)$ variable can be written as a scale mixture of $G(k, \mu)$ variables for integer $k>r$. To the best of our knowledge, this is a new result. The explicit form of the mixture is

$$
\begin{aligned}
\frac{\theta^{r} x^{r-1} \exp (-\theta x)}{\Gamma(r)}= & \int_{\theta}^{\infty}\left(\frac{\theta^{r} \Gamma(k) \mu^{-k}(\mu-\theta)^{k-r-1}}{\Gamma(r) \Gamma(k-r)}\right) \\
& \times \Gamma(k)^{-1} \mu^{k} x^{k-1} \exp (-\mu x) d \mu .
\end{aligned}
$$

The mixing density is the bracketed term in (5) and is the density of the scale parameter $\mu$ in the region $\mu \geq \theta$. Equation (5) can be verified by performing the integration. Note that when $r$ is already an integer (as in the case of $\lambda_{m}$ for $(m, m)$ systems) the gamma process is already $\chi^{2}$, and we can use $\chi^{2}$ results directly without mixing.

Let $X(t)=\sum_{i=1}^{k} g_{i}^{2}(t)$ be a $\chi^{2}$ process driven by $k$ independent zero mean Gaussian processes $g_{1}(t), g_{2}(t), \ldots, g_{k}(t)$ with common ACF $\mathrm{E}\left\{g_{i}(t) g_{i}(t+\tau)\right\}=\sigma^{2} R(\tau)$. Then, the LCR across a threshold $T$ is given by [11]

$\operatorname{LCR}_{\chi^{2}}(T)=\pi^{-1 / 2} \Gamma(k)^{-1} \sqrt{-R^{\prime \prime}(0)}(\mu T)^{k-1 / 2} \exp (-\mu T)$

for $T \geq 0$ and $\mu=\left(2 \sigma^{2}\right)^{-1}$ to agree with (5). Hence, if we construct a gamma process from a $\chi^{2}$ process as in (5), the LCR is also a mixture of $\chi^{2}$ LCRs as below:

$$
\begin{aligned}
\operatorname{LCR}_{G}(T)= & \int_{\theta}^{\infty}\left(\frac{\theta^{r} \Gamma(k) \mu^{-k}(\mu-\theta)^{k-r-1}}{\Gamma(r) \Gamma(k-r)}\right) \\
& \times \frac{\sqrt{-R^{\prime \prime}(0)}(\mu T)^{k-1 / 2} \exp (-\mu T)}{\sqrt{\pi} \Gamma(r)} d \mu .
\end{aligned}
$$


After a little algebra, the integral in (7) becomes [13]

$$
\begin{aligned}
\operatorname{LCR}_{G}(T)= & \frac{(\theta T)^{k-1 / 2} \sqrt{-R^{\prime \prime}(0)} \exp (-\theta T)}{\sqrt{\pi} \Gamma(r)} \\
& \times \Phi(k-r, k-r+1 / 2, \theta T)
\end{aligned}
$$

where $\Phi(a, b, c)$ is the confluent hypergeometric function. Equation (8) is our approximate LCR for each eigenvalue process. To implement (8), we must specify the parameters $\left(k, R^{\prime \prime}(0), \theta, r, T\right)$. Parameters $(r, \theta)$ are found using the gamma approximation for each eigenvalue $\lambda_{i}$ with $\theta=$ $\mathrm{E}\left\{\lambda_{i}\right\} / \operatorname{var}\left(\lambda_{i}\right)$ and $r=\theta \mathrm{E}\left\{\lambda_{i}\right\}$. The parameter $T$ is simply the level crossing threshold, and we choose $k$ as the smallest integer greater than or equal to $r$. The only parameter remaining is $R^{\prime \prime}(0)$, which we now consider.

\section{A. Eigenvalue Autocorrelation}

It is awkward to evaluate $R^{\prime \prime}(0)$ since $R(\tau)$ is the ACF of the Gaussians driving the $\chi^{2}$ process. Because we have constructed an infinite mixture of $\chi^{2}$ processes to approximate the eigenvalue process, these underlying Gaussians are fictitious, having no physical meaning. However, whatever ACF they have must lead to the correct ACF for the eigenvalues themselves. Hence, we work backwards, evaluating the eigenvalue $\mathrm{ACF}$ and selecting $R(\tau)$ to result in this value. Note that the ACF we consider is the normalized version (correlation coefficient) to match the results in [11].

The eigenvalue ACF when the $\boldsymbol{H}(t)$ matrix is driven by a Jakes process appears to be unknown. However, we only require $R(\tau)$ at $\tau \approx 0$, so it suffices to compute the eigenvalue $\mathrm{ACF}$ at lags close to zero. In [6] a stochastic differential equation is derived for the eigenvalues $\boldsymbol{w}=\left(w_{1}, w_{2}, \ldots, w_{m}\right)$ of $\boldsymbol{H}_{B} \boldsymbol{H}_{B}^{T}$ when the elements of $\boldsymbol{H}_{B}: m \times n$ follow i.i.d. real standard Brownian motion processes and $m, n$ are as defined in Section II. This result also applies to the complex case when $\mathrm{E}\left\{\left|\left[\boldsymbol{H}_{B}\right]_{i j}\right|^{2}\right\}=1$, where $[\boldsymbol{A}]_{i j}$ is the $(i, j)$-th element of matrix $\boldsymbol{A}$. Hence, we have the stochastic differential equation [6]

$$
d w_{i}(t)=2 \sqrt{w_{i}(t)} d B_{i}(t)+n d t+\sum_{k \neq i} \frac{w_{i}(t)+w_{k}(t)}{w_{i}(t)-w_{k}(t)} d t
$$

where the $w_{i}(t)$ are the time varying eigenvalues of $\boldsymbol{H}_{B}(t)$ and $B_{i}(t)$ is the driving Brownian motion process, which is independent of $w_{j}(t)$ and $B_{j}(t)$ for $i \neq j$. Using the standard Euler approximation [14] to (9), we have for small $\tau>0$

$$
\begin{aligned}
w_{i}(t+\tau) \approx & w_{i}(t)+2 \sqrt{w_{i}(t)}\left[B_{i}(t+\tau)-B_{i}(t)\right]+n \tau \\
& +\tau \sum_{k \neq i} \frac{w_{i}(t)+w_{k}(t)}{w_{i}(t)-w_{k}(t)}
\end{aligned}
$$

Multiplying by $w_{i}(t)$ and taking expectations gives

$$
\begin{aligned}
C_{w_{i}}(\tau) & \approx C_{w_{i}}(0) \\
& +\tau \mathrm{E}\left\{n w_{i}(t)+w_{i}(t) \sum_{k \neq i} \frac{w_{i}(t)+w_{k}(t)}{w_{i}(t)-w_{k}(t)}\right\}
\end{aligned}
$$

where $C_{w_{i}}(\tau)=\mathrm{E}\left(w_{i}(t) w_{i}(t+\tau)\right)$.

To use (10) and (11), we need to transform $\boldsymbol{H}_{B}(t)$ and $\boldsymbol{H}_{B}(t+\tau)$ so they have the same joint distribution as $\boldsymbol{H}(t)$
TABLE III

K VALUES

\begin{tabular}{c|cccc}
\hline System & $\lambda_{1}$ & $\lambda_{2}$ & $\lambda_{3}$ & $\lambda_{4}$ \\
\hline \hline$(2,2)$ & -0.07692 & -1 & & \\
\hline$(2,4)$ & -0.1453 & -0.7643 & & \\
\hline$(3,3)$ & -0.1827 & -0.8849 & -2 & \\
\hline$(4,4)$ & -0.2792 & -0.9644 & -1.6312 & -3 \\
\hline
\end{tabular}

and $\boldsymbol{H}(t+\tau)$ under the desired Jakes model. Once this is done, Equation (10) becomes a difference equation for the eigenvalue $\lambda_{i}(t)$ as required. Since the entries of $\boldsymbol{H}_{B}(t)$ are complex standard Brownian motion we have $\mathrm{E}\left\{\left[\boldsymbol{H}_{B}(t)\right]_{i j}\right\}=$ $0, \mathrm{E}\left\{\left|\left[\boldsymbol{H}_{B}(t)\right]_{i j}\right|^{2}\right\}=t$ and $\mathrm{E}\left\{\left(\left[\boldsymbol{H}_{B}(t)\right]_{i j}\left[\boldsymbol{H}_{B}^{*}(t+\tau)\right]_{i j}\right)\right\}=$ $t$. The $\boldsymbol{H}(t)$ matrix, on the other hand, has elements with zero mean, magnitude variance equal to 1 and covariance equal to $J_{0}\left(2 \pi f_{D} \tau\right)$. Note that $\left[t^{-1 / 2} \boldsymbol{H}_{B}(t)\right]_{i j}$ has the correct mean, variance and covariance function equal to $\sqrt{t /(t+\tau)}$. If we consider the particular time point $t=\tau\left[J_{0}\left(2 \pi f_{D} \tau\right)^{-2}-1\right]^{-1}$, then $t^{-1 / 2}\left[\boldsymbol{H}_{B}(t)\right]_{i j}$ has the correct covariance function and exactly the same joint distribution at $(t, t+\tau)$ as the desired process $[\boldsymbol{H}(t)]_{i j}$. Hence, we rewrite (10) using this particular time value and replace $w_{i}(t)$ by $t \lambda_{i}(t)$, noting that $w_{i}(t)$ and $t \lambda_{i}(t)$ are statistically identical at $t$ and $t+\tau$

$$
\begin{aligned}
t(t+\tau) & C_{\lambda_{i}}(\tau) \approx t^{2} C_{\lambda_{i}}(0) \\
& +\tau t \mathrm{E}\left\{n \lambda_{i}(t)+\lambda_{i}(t) \sum_{k \neq i} \frac{\lambda_{i}(t)+\lambda_{k}(t)}{\lambda_{i}(t)-\lambda_{k}(t)}\right\} .
\end{aligned}
$$

Next, we standardise $C_{\lambda_{i}}(\tau)$ to give the ACF and expand (12) in powers of $\tau$. After a little algebra and inserting the particular value of $t$, we find

$$
R_{\lambda_{i}}(\tau)=1+2 \pi^{2} f_{D}^{2} \tau^{2}(K-1)+o\left(\tau^{3}\right)
$$

where

$$
\begin{aligned}
K \operatorname{var}\left(\lambda_{i}(t)\right)= & \mathrm{E}\left\{n \lambda_{i}(t)+\lambda_{i}(t) \sum_{k \neq i} \frac{\lambda_{i}(t)+\lambda_{k}(t)}{\lambda_{i}(t)-\lambda_{k}(t)}\right\} \\
& -C_{\lambda_{i}}(0) .
\end{aligned}
$$

Note that $K$ in (14) is defined in terms of the first and second moments of $\lambda_{i}(t)$ and the mean of the summation term. The first two moments are given in Tables I and II. The expected value of the summation in (14) appears more complex, but exact calculations are possible. For reasons of space we omit the details, but it can be seen that the expected value involves multiplying the summation term by the joint density (2). Hence, the denominator cancels out, and the subsequent integration is only over polynomial and exponential terms. Using a symbolic software package, we have calculated $K$ for $(2,2),(2,4),(3,3)$ and $(4,4)$ systems as shown in Table III.

We now use the eigenvalue ACF (13) for small lags to derive $R(\tau)$. The gamma process approximation must have the correct ACF (13). This can be achieved by all the $\chi^{2}$ processes in the infinite mixture having the same ACF. Hence, we choose the ACF $R(\tau)$ for each underlying Gaussian component $g_{i}(t)$ to produce a $\chi^{2}$ process with ACF (13). Calculations show that the $\chi^{2}$ process has ACF $R^{2}(\tau)$ when 
driven by a Gaussian process with $\mathrm{ACF} R(\tau)$. Therefore, we require $R(\tau)=R_{\lambda_{i}}(\tau)^{1 / 2}$. For small $\tau$ this gives

$$
R(\tau)=1+\pi^{2} f_{D}^{2} \tau^{2}(K-1)+o\left(\tau^{3}\right)
$$

and so the second derivative at zero is

$$
R^{\prime \prime}(0)=2 \pi^{2} f_{D}^{2}(K-1) .
$$

\section{B. Final LCR and AFD Approximations}

Substituting (16) into (8) gives the final LCR approximation

$$
\begin{aligned}
\operatorname{LCR}_{G}(T)= & \frac{(\theta T)^{k-1 / 2} \pi f_{D} \sqrt{2(1-K)} \exp (-\theta T)}{\sqrt{\pi} \Gamma(r)} \\
& \times \Phi(k-r, k-r+1 / 2, \theta T) .
\end{aligned}
$$

The AFD follows from the result [15]

$$
\operatorname{AFD}_{\lambda_{i}}(T)=F_{\lambda_{i}}(T) / \operatorname{LCR}_{\lambda_{i}}(T) \text {. }
$$

As discussed in the introduction, the distribution $F_{\lambda_{i}}(T)$ can be computed exactly, and so (18) is a closed form approximation.

\section{Computer Simulations}

To verify our analytical results, we performed Monte Carlo simulations using the improved Jakes' simulator of [16] to generate the $\boldsymbol{H}(t)$ matrix. Simulations of the LCRs and AFDs were performed for all systems with $2 \leq m, n \leq 4$. A selection of these cases is shown in Figs. $2-6$. The approximate LCRs and AFDs were calculated from (17) and (18). We see that agreement is excellent.

Although the gamma approximations for the LCRs, AFDs and CDFs accurately track the simulation results, close inspection shows that the gamma fit deteriorates as $m$ increases or as $n-m$ increases. Based on preliminary simulation results, one solution is to model the eigenvalues as a mixture of two separate gamma components. Since LCRs are known for both gamma components, the overall LCR is also readily available, but is beyond the scope of this paper.

From Figs. $2-6$ we draw the following conclusions. As expected, the width of the LCR curves and the peak threshold increase from $\lambda_{\min }$ to $\lambda_{\max }$, since the mean and variances also increase. Comparing $(2,4)$ with $(2,2)$, we see the effect of the extra diversity which has a smoothing effect yielding LCR curves that are narrower for the $(2,4)$ case and peak at higher thresholds. In all cases, the peak LCR drops from $\lambda_{\min }$ to $\lambda_{\max }$ but only marginally. The smaller eigenvalues, especially $\lambda_{\min }$, experience deep fades quite often, but their AFDs are short. At the other extreme, $\lambda_{\max }$ corresponds to the eigenchannel carrying the largest constellations in adaptive modulation. Here, for large thresholds the AFDs are long and the LCRs are low. Hence, depending on $f_{D}$, it may be possible to select thresholds to give a reasonable adaptation rate for all eigenchannels.

\section{CONCLUSIONS}

In this paper we have studied the time-variations of the channel in MIMO systems. To help characterize the performance of adaptive MIMO systems using, for example, SVD transmission or channel inversion, we have analyzed the LCRs and AFDs of the channel eigenvalues. Using a gamma process approximation to the eigenvalues, we have derived simple but elegant closed-form approximations for the LCRs and AFDs. Finally, for large systems we have discussed an approach to further improve the accuracy.

\section{REFERENCES}

[1] I. E. Telatar, "Capacity of multi-antenna Gaussian channels," European Trans. on Telecomm. Related Technol., vol. 10, pp. 585-595, Nov.-Dec. 1999.

[2] G. Lebrun, T. Ying, and M. Faulkner, "MIMO transmission over a timevarying channel using SVD," in Proc. 2002 IEEE Global Telecommunications Conf., Taipei, Taiwan, Nov. 17-21, 2002, pp. 414-418.

[3] A. J. Goldsmith and S.-G. Chua, "Adaptive coded modulation for fading channels," IEEE Trans. Commun., vol. 46, no. 5, pp. 595-602, May 1998.

[4] C. B. Peel, B. M. Hochwald, and A. L. Swindlehurst, "A vectorperturbation technique for near-capacity multi-antenna multi-user communication - Part I: Channel inversion and regularization," submitted to IEEE Trans. Commun., June 2003.

[5] A. Abdi, C. Gao, and A. M. Haimovich, "Level crossing rate and average fade duration in MIMO mobile fading channels," in Proc. 58th IEEE Vehicular Technology Conf., VTC Fall 2003, Orlando, Florida, USA, Oct. 6-9, 2003, pp. 3164-3168.

[6] M. F. Bru, "Diffusions of perturbed principal component analysis," $J$. Multivariate Anal., vol. 29, pp. 127-136, 1989.

[7] P. J. Smith and L. M. Garth, "Distribution and characteristic functions of correlated complex Wishart matrices," submitted to J. Multivariate Anal., Feb. 2004.

[8] J. T. Y. Ho and P. J. Smith, "A quick simulation method for fading communications channels using a novel eigenvalue importance sampling technique," in Proc. 56th IEEE Vehicular Technology Conf., VTC Fall 2002, Vancouver, BC, Sept. 24-28, 2002, pp. 449-453.

[9] A. Edelman, "Eigenvalues and condition numbers of random matrices," Ph.D. dissertation, MIT, 1989.

[10] S. R. Adke and N. Balakrishna, "Markovian chi-square and gamma processes," Statist. Probab. Lett., vol. 15, no. 5, pp. 349-356, Dec. 1992.

[11] R. A. Silverman, "The fluctuation rate of the chi process," IRE Trans. Inform. Theory, vol. 4, no. 1, pp. 30-34, Mar. 1958.

[12] L. J. Gleser, "The gamma distribution as a mixture of exponential distributions," Amer. Statist., vol. 43, no. 2, pp. 115-117, May 1989.

[13] I. S. Gradshteyn and I. M. Ryzhik, Table of Integrals, Series, and Products, 6th ed. San Diego: Academic Press, 2000.

[14] T. Mikosch, Elementary Stochastic Calculus with Finance in View. River Edge, NJ: World Scientific Publ., 1998.

[15] W. C. Jakes, Ed., Microwave Mobile Communications. Piscataway, NJ: IEEE Press Classic Reissue, 1995.

[16] Y. Li and X. Huang, "The simulation of independent Rayleigh faders," IEEE Trans. Commun., vol. 50, no. 9, pp. 1503-1514, Sept. 2002. 


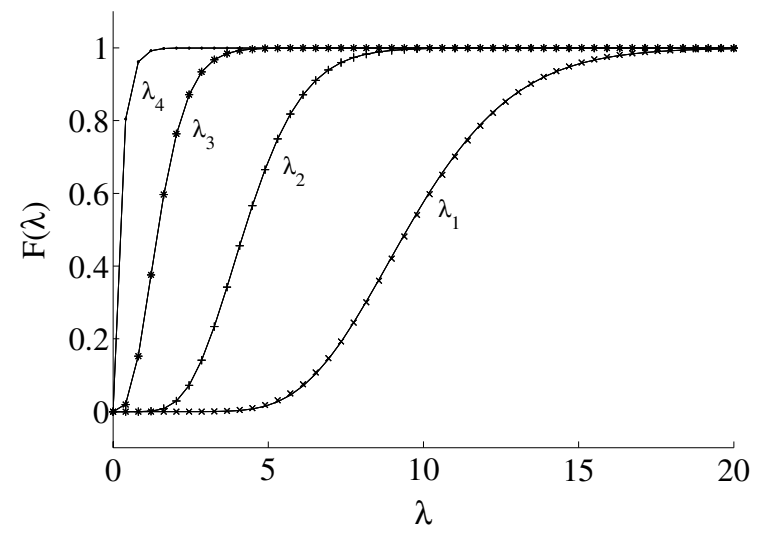

Fig. 1. CDFs for the eigenvalues of a MIMO $(4,4)$ system. Points Fig. 2. LCRs for the eigenvalues of a MIMO $(4,4)$ system. denote the Gamma CDF, while lines denote the exact eigenvalue CDF. Points represent simulated results and lines represent the analytical

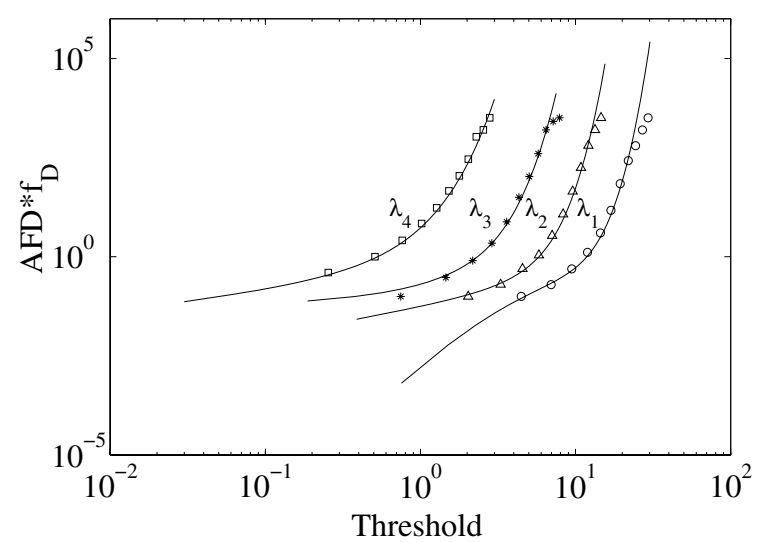

Fig. 3. AFDs for the eigenvalues of a MIMO $(4,4)$ system.
Points represent simulated results and lines represent the analytical approximations.

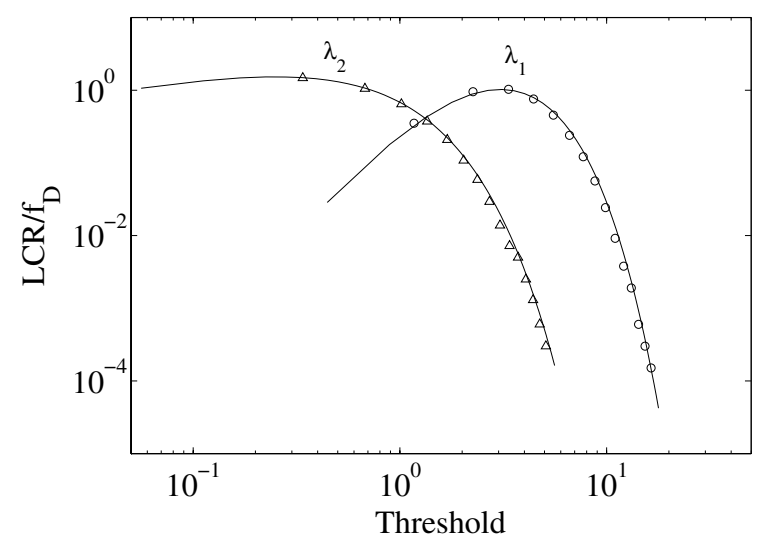

Fig. 5. LCRs for the eigenvalues of a MIMO (2,2) system. Points represent simulated results and lines represent the analytical approximations. approximations.
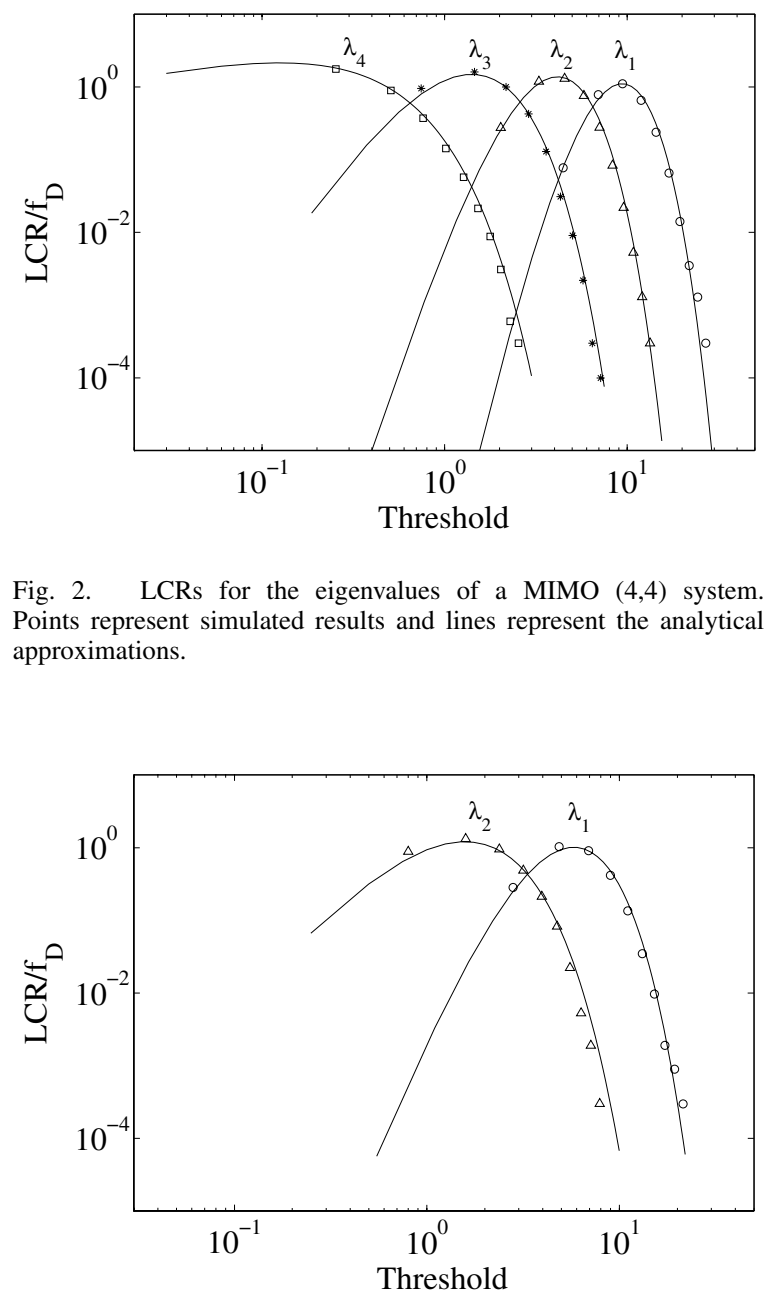

Fig. 4. LCRs for the eigenvalues of a MIMO $(2,4)$ system Points represent simulated results and lines represent the analytical approximations.

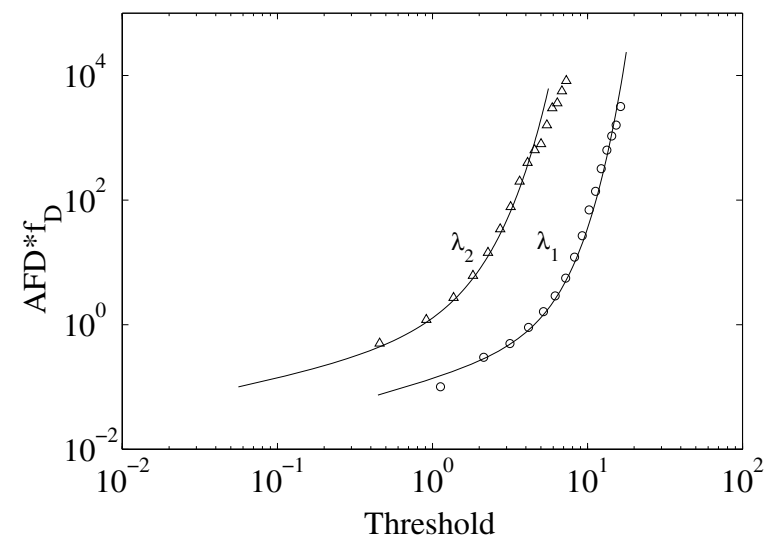

Fig. 6. AFDs for the eigenvalues of a MIMO $(2,2)$ system. Points represent simulated results and lines represent the analytical approximations. 